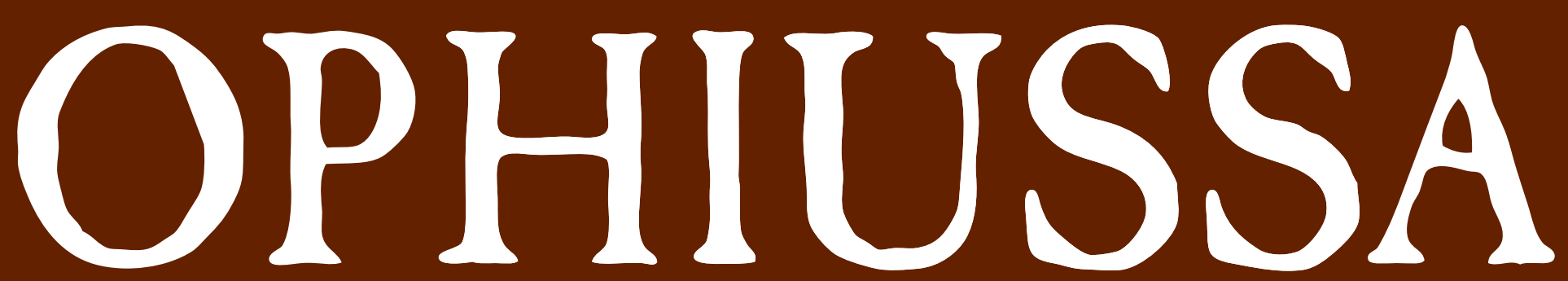

REVISTA DO CENTRO DE ARQUEOLOGIA DA UNIVERSIDADE DE LISBOA

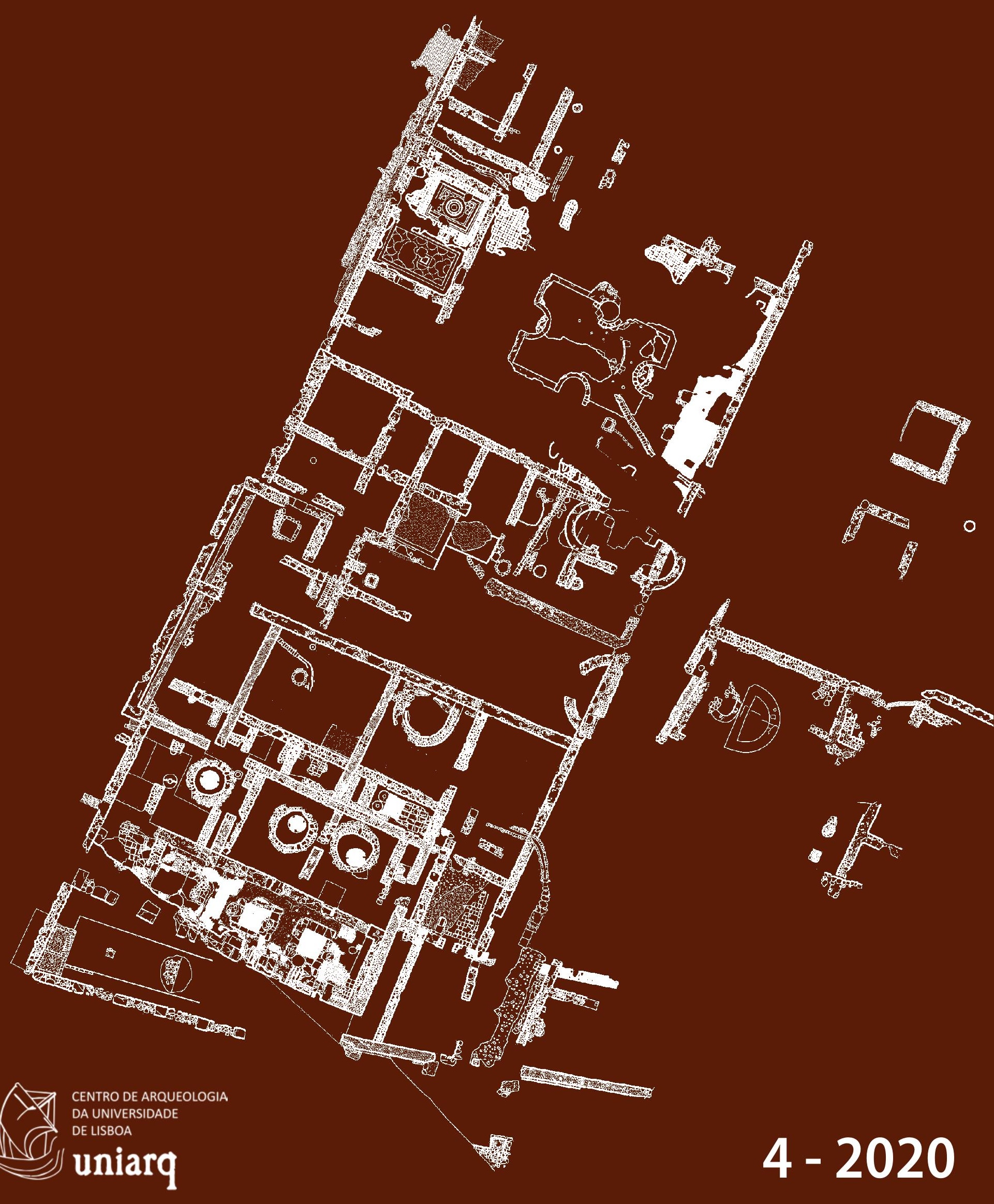




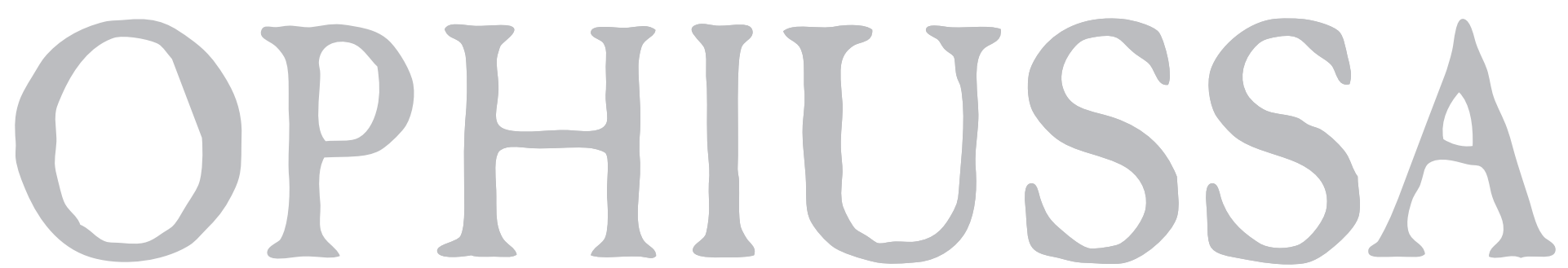

REVISTA DO CENTRO DE ARQUEOLOGIA DA UNIVERSIDADE DE LISBOA

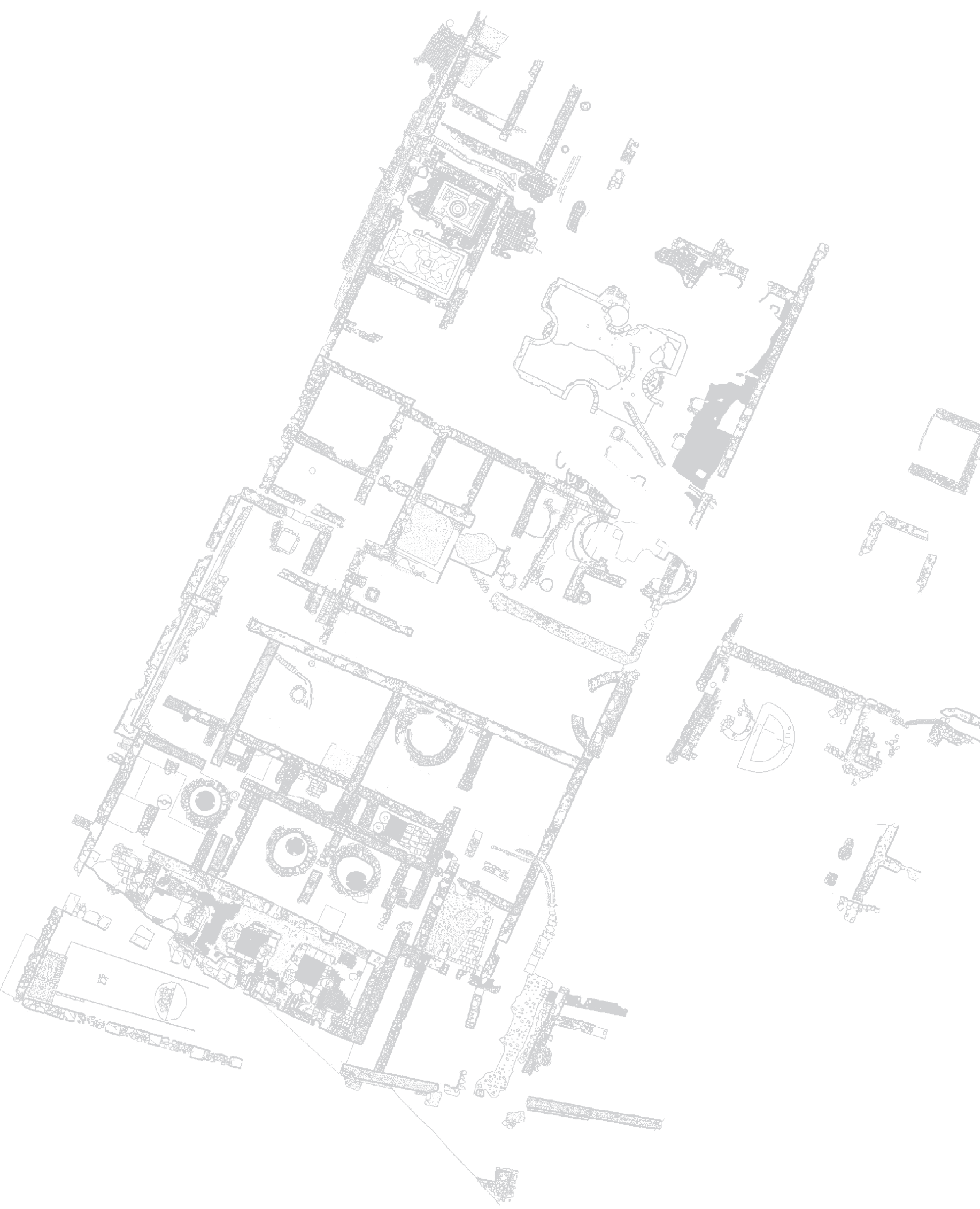




\section{U IISBOA \\ UNIVERSIDADE

\section{OPHIUSSA REVISTA DO CENTRO DE ARQUEOLOGIA DA UNIVERSIDADE DE LISBOA}

PUBLICAÇÃO ANUAL · ISSN 1645-653X · E-ISSN 2184-173X

Volume 4 - 2020

DIRECÇÃO E COORDENAÇÃO EDITORIAL

Ana Catarina Sousa

Elisa Sousa

\section{CONSELHO CIENTÍFICO}

André Teixeira

UNIVERSIDADE NOVA DE LISBOA

Carlos Fabião

UNIVERSIDADE DE LISBOA

Catarina Viegas

UNIVERSIDADE DE LISBOA

Gloria Mora

UNIVERSIDAD AUTÓNOMA DE MADRID

Grégor Marchand

CENTRE NATIONAL DE LA RECHERCHE SCIENTIFIQUE

João Pedro Bernardes

UNIVERSIDADE DO ALGARVE

José Remesal

UNIVERSIDADE DE BARCELONA

Leonor Rocha

UNIVERSIDADE DE ÉVORA

Manuela Martins

UNIVERSIDADE DO MINHO

Maria Barroso Gonçalves

INSTITUTO SUPERIOR DE CIENNCIAS DO TRABALHO E DA EMPRESA)

Mariana Diniz

UNIVERSIDADE DE LISBOA

Raquel Vilaça

UNIVERSIDADE DE COIMBRA

Victor S. Gonçalves

UNIVERSIDADE DE LISBOA

Xavier Terradas Battle

CONSEJO SUPERIOR DE INVESTIGACIONES CIENTÍFICAS

\section{SECRETARIADO}

André Pereira

CAPA

Julia Rodríguez Aguilera

(Gespad al Andalus)
REVISOR DE ESTILO

Francisco B. Gomes

PAGINAÇÃo

TVM Designers

IMPRESSÃO

AGIR - Produções Gráficas

DATA DE IMPRESSÃO

Dezembro de 2020

EDIÇÃO IMPRESSA (PRETO E BRANCO)

300 exemplares

EDIÇÃO DIGITAL (A CORES)

www.ophiussa.letras.ulisboa.pt

ISSN 1645-653X / E-ISSN 2184-173X

DEPÓSITO LEGAL 190404/03

Copyright $\odot 2020$, os autores

EDIÇÃO

UNIARQ - Centro de Arqueologia

da Universidade de Lisboa,

Faculdade de Letras de Lisboa

1600-214 Lisboa.

www.uniarq.net

www.ophiussa.letras.ulisboa.pt

uniarq@letras.ulisboa.pt

Revista fundada por Victor S. Gonçalves (1996). O cumprimento do acordo ortográfico de 1990 foi opção de cada autor.

Esta publicação é financiada por fundos nacionais através da FCT - Fundação para a Ciência e a Tecnologia, I.P., no âmbito do projecto UIDB/00698/2020. 
ÍNDICE

The megalithic clusters of Deserto and Barrocal das Freiras

(Montemor-o-Novo, Middle Alentejo) in the building of the sacred

landscapes of ancient peasant societies of the $4^{\text {th }}$ and $3^{\text {rd }}$ millennia BCE

VICTOR S. GONÇALVES, MARCO ANTÓNIO ANDRADE

From aDNA to Archaeology: Genética da transição Calcolítico-Idade do Bronze

no Sul de Portugal

ANA CATARINA BASÍLIO

Foundry in the Late Bronze Age Baiões/Santa Luzia Cultural Group:

some reflections starting from a new metallic mould for unifacial palstaves

JOÃO CARLOS SENNA-MARTINEZ, PEDRO VALÉRIO, MARIA HELENA CASIMIRO,

LUÍS M. FERREIRA, MARIA DE FÁTIMA ARAÚJO, HORÁCIO PEIXOTO

O conjunto vítreo da necrópole da I Idade do Ferro da Fonte Velha

de Bensafrim (Lagos)

FRANCISCO B. GOMES

Espacios de almacenamiento y producción de la Turdetania.

Una reflexión más allá de los hornos

VIOLETA MORENO MEGÍAS

Os três sarcófagos etruscos da colecção de Sir Francis Cook

no Museu Arqueológico de São Miguel de Odrinhas (Sintra)

MARTA RIBEIRO, NUNO SIMÕES RODRIGUES

Nuevos datos para el conocimiento del suburbium del Municipum

Florentinum Iliberritanum (Granada): las recientes intervenciones

en el solar de Mondragones

ÁNGEL RODRÍGUEZ AGUILERA, MACARENA BUSTAMANTE-ÁLVAREZ,

JULIA RODRÍGUEZ AGUILERA, CARMEN JÓDAR HÓDAR, JOSÉ M. GARCÍA-CONSUEGRA FLORES

Mapear a bibliografia. Abordagem metodológica para a gestão

de dados bibliográficos

NATÁLIA BOTICA, SÍLVIA MACIEL, REBECA BLANCO-ROTEA

Recensões bibliográficas

(TEXTOS: JORGE DEL REGUERO GONZÁLEZ, LUIS MIGUEL CARRANZA PECO,

MARTA BERMÚDEZ CORDERO, ÁLVARO GÓMEZ PEÑA, ANDRÉ TEXUGO)

Da CAALG à UNIARQ: a génese do Centro de Arqueologia da Universidade

de Lisboa no quadro do sistema científico de meados dos anos 70

a meados dos anos 90 do século XX

ANDRÉ PEREIRA

Avaliadores Ophiussa (2018-2020) 


\title{
Mapear a bibliografia. Abordagem metodológica para a gestão de dados bibliográficos
}

\author{
Mapping bibliography. \\ A methodological approach for \\ bibliographic data management
}

\author{
NATÁLIA BOTICA \\ Universidade de Minho. Lab2PT \\ nb@uaum.uminho.pt \\ SÍLVIA MACIEL \\ Arqueóloga. \\ silvia13mariana@gmail.com \\ REBECA BLANCO-ROTEA \\ Universidade de Santiago de Compostela; Síncrisis. \\ rebeca.blanco.rotea@usc.es
}

RESUMO: A investigação em arqueologia parte sempre de uma procura documental e bibliográfica para o levantamento de dados e conhecimento já adquirido sobre a temática a estudar. Desta tarefa resulta uma grande quantidade de informação que, para casos de estudos macro e/ou semi-macro, como no caso do projeto de estudo da paisagem arqueológica da Citânia de Sanfins, implica uma estratégia para gestão desta informação. Assim, para este caso de estudo, usou-se o sistema de informação 2ArchIS para estruturar e normalizar os dados recolhidos e associá-los a metadados. Este sistema permitiu agilizar o processo de procura e de mapeamento de dados, quer na interrogação direta à base de dados, quer através do seu mapeamento num Sistema de Informação Geográfico, que interliga os (meta)dados associados a documentos e bibliografia aos sítios arqueológicos, e permite representar graficamente a informação qualitativa e quantitativa referente a cada sítio em estudo.

PALAVRAS-CHAVE: Arqueologia; Bibliografia; Historiografia; Base de Dados; SIG.

ABSTRACT: Archaeological research always starts from a documentary and bibliographic search to collect data and knowledge already acquired on the subject to be studied. This task results in a large amount of information that, for cases of macro and / or semi-macro studies, such as the study project of the archeological landscape of Citânia de Sanfins, demands a strategy for documentation management. Thus, for this case study, we used the information system 2 ArchlS to structure and normalize the compiled data and associate them with metadata. This system allowed us to optimize the search process of searching and mapping data, either in the direct interrogation of the database or through its mapping in a Geographic Information System that links documents and bibliography meta(data) to archaeological sites, allowing graphic representations of quantitative and qualitative data.

KEYWORDS: Archaeology; Bibliography; Historiography; Database; GIS. 


\section{INTRODUÇÃO}

A revisão de literatura e a pesquisa de dados, ponto de partida de qualquer investigação, pode-se configurar um processo não só desafiante, mas também complexo e moroso. $O$ enquadramento e a justificação da problemática em estudo são necessários para construir uma base sólida que permita explorar o tema em análise, pelo que é imprescindível que a informação recolhida seja tratada de forma estruturada e consistente.

O estudo da paisagem arqueológica da Citânia de Sanfins, para além da pesquisa documental e bibliográfica sobre os estudos realizados, implicou também fazer um levantamento sistemático de todos os trabaIhos e dados arqueológicos feitos na região.

$\mathrm{Na}$ área de arqueologia, o levantamento da informação dos trabalhos arqueológicos revela-se bastante difícil e demorado. O "Portal do Arqueólogo", da responsabilidade da Direção Geral do Património Cultural (DGPC) é a interface oficial para publicação online dos trabalhos arqueológicos em Portugal, mas é omisso para grande parte dos trabalhos já realizados. Assim, esta pesquisa é feita em artigos e relatórios publicados em formatos e suportes muito dispersos. Os dados resultantes dos trabalhos arqueológicos não são, na sua grande parte localizáveis e, quando o são, não estão acessíveis.

No decurso do desenvolvimento do estudo sobre a paisagem arqueológica da Citânia de Sanfins (Maciel 2019), esta dificuldade foi particularmente sentida pelo facto de o mesmo implicar a análise e correlação da informação existente, relativa a uma zona de estudo que envolveu mais do que um sítio arqueológico, tornando-se desafiante a agregação da informação dispersa, de forma a contextualizar e caracterizar devidamente os sítios em análise.

Neste contexto, consideramos pertinente explorar a questão da gestão de dados bibliográficos, implementando uma metodologia para apoiar o registo e tratamento da informação recolhida, cujos resultados permitem a sua utilização e visualização de modo mais sistemático e eficiente. Num trabalho futuro, abordaremos de uma forma mais promissora o potencial desta metodologia para a investigação e a gestão do património.

\section{ANÁLISE MACRO ESPACIAL DA CITÂNIA DE SANFINS}

A Citânia de Sanfins apresenta-se como um dos povoados mais excecionais do Noroeste da Península Ibérica, tendo despertado interesse em proceder à análise da respetiva paisagem, com vista a compreender dinâmicas políticas, sociais, económicas e simbólicas, que decorreram no território onde este povoado se integra, no final da Idade do Ferro e início do período Romano.

Considerando a centralidade da Citânia de Sanfins e a sua importância na administração do território em análise (Silva 2007), definiu-se como área de incidência do estudo a zona geográfica que se localiza no distrito do Porto, limitada a norte pelo rio Ave e a sul pelo rio Leça (Fig. 1). Esta área concentra um total de dezassete povoados, tendo sido necessário recolher os dados relativos a cada povoado de forma a caraterizar cronológica e culturalmente cada um deles. No conjunto de povoados que integram o estudo destacamos a relevância do castro de Guifões, em Matosinhos (Cleto - Varela 1999; Arezes - Varela 2017), o castro de AlvareIhos, na Trofa (Moreira 2009; Pinho 2009) e o castro de Monte Padrão em Santo Tirso (Martins 1985; Moreira 2005) não só pela sua longa ocupação, mas também pelo seu papel ativo durante a ocupação romana do território.

Com o objetivo de ensaiar possíveis relações dos povoados entre si e com a paisagem envolvente, executámos algumas análises geoespaciais, com base

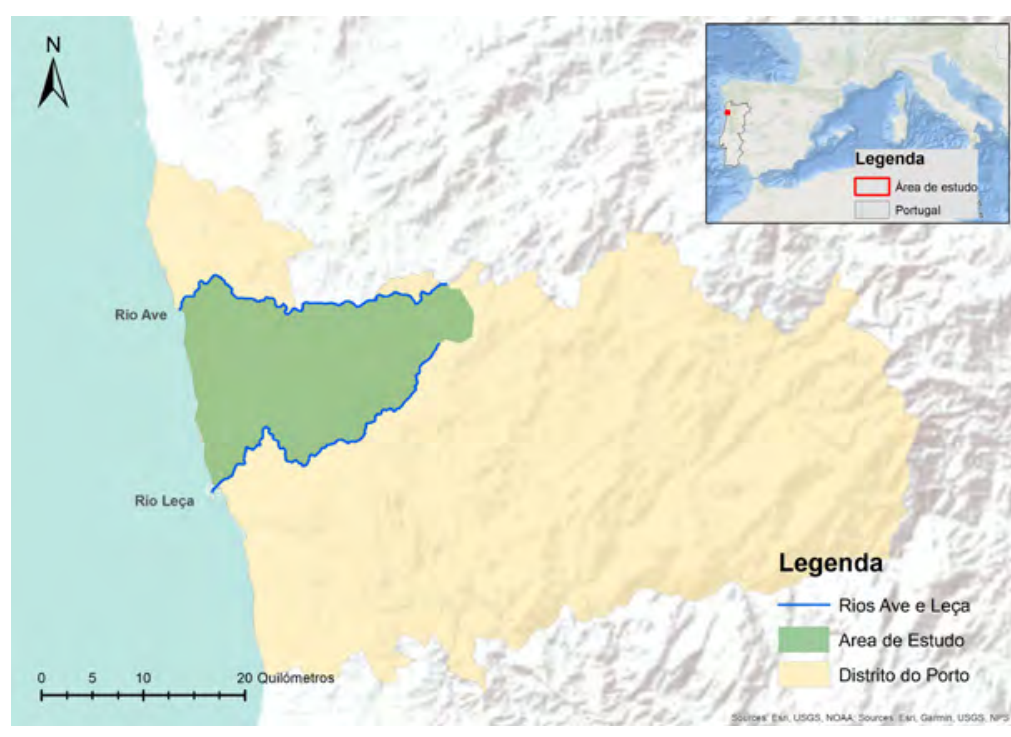

FIG. 1 Zona de estudo delimitada pelos rios Ave e Leça. 
em fatores como a altitude, a aptidão agrícola dos solos, a acessibilidade e a mobilidade (Maciel 2019). No entanto, a incorporação de informação disponível sobre os sítios em análise era também uma componente fundamental nesse processo. Iniciou-se a recoIha de informação dos sítios arqueológicos no "Portal do Arqueólogo", complementando com a documentação e bibliografia disponível, nomeadamente em dissertações de mestrado e teses de doutoramento. Muitos dos sítios foram intervencionados no século passado, sem informação disponível em suporte digital e, muita informação disponibilizada online era de acesso restrito ou em links para referências web que já não estão disponíveis. Para a maioria dos documentos encontrados foi evidente a ausência de metadados associados, o que dificultou muito a sua localização, para além da total ausência de acesso a dados abertos.

Estas questões e dificuldades são transversais a qualquer investigação em arqueologia e, o sistema 2ArchIS utilizado já noutros trabalhos (Botica - Andrade - Fontes 2016), revela-se uma ferramenta de gestão muito importante no apoio à investigação, reforçando a necessidade e vantagens inerentes a uma estrutura de dados e metadados consistentes, normalizados, acessíveis e interoperáveis (Botica et al. 2021).

\section{LOCALIZAÇÃO E ACESSO A DADOS ARQUEOLÓGICOS}

Tendo em conta as dificuldades sentidas na pesquisa de informação, acima referidas, direcionamos a nossa atenção para os diversos sistemas de informação criados em Portugal, ao longo dos últimos quarenta anos.

Na década de 80 do século passado, partindo da necessidade de constituir um inventário dos sítios arqueológicos identificados até ao momento, realizou-se o primeiro trabalho de inventariação de sítios arqueológicos em território nacional. Este primeiro sistema aglutinava informações de natureza técnica, científica, legal e administrativa' sendo que, apenas nos finais de 1995, se constituiu um Sistema

Divisão de Inventário do Instituto Português de Arqueologia 2002. de Informação e Gestão Arqueológica designado por "Endovélico", sistema que alimenta o atual "Portal do Arqueólogo"2.

Os responsáveis pelos trabalhos arqueológicos sentiram também eles a necessidade de organizarem o crescente volume e diversidade de dados resultantes desses trabalhos. Assim, desde muito cedo começaram a recorrer a tecnologias de informação para registo de dados, tendo sido criado um cenário de grande variedade de sistemas e formatos, alojados nos computadores pessoais dos arqueólogos ou em servidores institucionais. Um exemplo desse esforço é o sistema de informação estruturado pela Unidade de Arqueologia da Universidade do Minho, já na década de 90 do século passado, que permitiu a gestão do grande volume de dados das intervenções arqueológicas realizadas em Braga no âmbito do «Projeto de Salvamento de Bracara Augusta» (Botica - Martins 2008).

No entanto, apesar das diversas iniciativas de gestão da informação arqueológica, na grande maioria não obedecem a esquemas de dados padrão nem a uma uniformização de terminologias, pelo que, quando se tem acesso a esses dados continuamos a sentir dificuldades no processo de integração da informação, como aponta M. Almeida aquando do desenvolvimento do seu projeto de doutoramento (Almeida 2017a), que se deparou com a heterogeneidade de informação, a qual causa obstáculos ao tratamento integrado e à normalização da mesma, dificultando a sua reutilização (Almeida 2017b: 168).

A temática da dispersão de dados arqueológicos, da falta de repositórios e da dificuldade no acesso e reutilização, tem sido objeto de reflexões de toda a comunidade científica em geral e da Arqueologia em particular, uma vez que as escavações arqueológicas são ações irrepetíveis e, em caso de perda, os dados são irrecuperáveis.

Se, por um lado os arquivos em papel são dificilmente localizáveis e acessíveis, por outro, os registos digitais, quando não guardados em repositórios perenes, correm risco de desparecer ou de obsolescência, quer pelos suportes onde são guardados, quer pelos formatos de ficheiros que são utilizados. Alguns projetos internacionais foram lançados para definir

http://arqueologia.patrimoniocultural.pt/ 
estratégias e procedimentos comuns sobre as boas práticas de gestão e salvaguarda de dados. Destacamos o projeto ARIADNE (Niccolucci - Richards 2013) e o seu sucessor ARIADNEPlus, um dos maiores projetos internacionais que envolve 23 países, sendo a UAUM um parceiro associado, que tem como principal objetivo a criação de uma infraestrutura europeia de pesquisa on-line para dados arqueológicos. No entanto, este projeto deparou-se com a falta de equidade dos países participantes, onde apenas três dos países, ADS (Reino Unido), DANS (Países Baixos) e SND (Suécia), têm repositórios nacionais para dados arqueológicos. Para os restantes países os dados estão dispersos por arquivos individuais e institucionais, que espelham diferentes escolas e práticas em arqueologia, diferentes condicionalismos legais e diferentes capacidades de preparação e gestão de dados. Os projetos internacionais SEADDA (Richards - Wright 2019), onde a UAUM é membro, e o projeto Parthenos (Hollander et al. 2019), têm como principal objetivo criar entendimentos comuns sobre as melhores práticas internacionais para a preservação, disseminação e reutilização de dados arqueológicos e a sua interoperabilidade com infraestruturas, como a criada pelo projeto ARIADNE.

\section{METODOLOGIA}

O projeto «A paisagem na Idade do Ferro e Romanização da Citânia de Sanfins, Paços de Ferreira. Análise macro espacial» envolve o estudo e análise de um conjunto de dados arqueológicos associados aos sítios, nomeadamente a sua tipologia, períodos cronológicos, intervenções realizadas e espólio associado, mas também informação relativa ao contexto geológico, hidrográfico e de uso de solo, bem como um vasto tipo de mapas e cartografia. Para gestão de dados e de formatos tão distintos foi usado um sistema de gestão de dados arqueológicos e de contexto, interligando-os com a documentação e bibliografia identificadas (Fig. 2).

Todos os registos da Base de Dados estão interligados entre si, tendo-se procedido à normalização dos descritores e à associação de metadados que nos irão permitir estabelecer critérios de pesquisa e de seleção sobre os dados. Relativamente à bibliografia, para além dos descritores standard de autor, publicação, data, etc, foram ainda associados metadados relativos aos conteúdos, nomeadamente aos sítios arqueológicos, aos materiais de escavações arqueológicas realizadas ou a estudos e temas abordados.

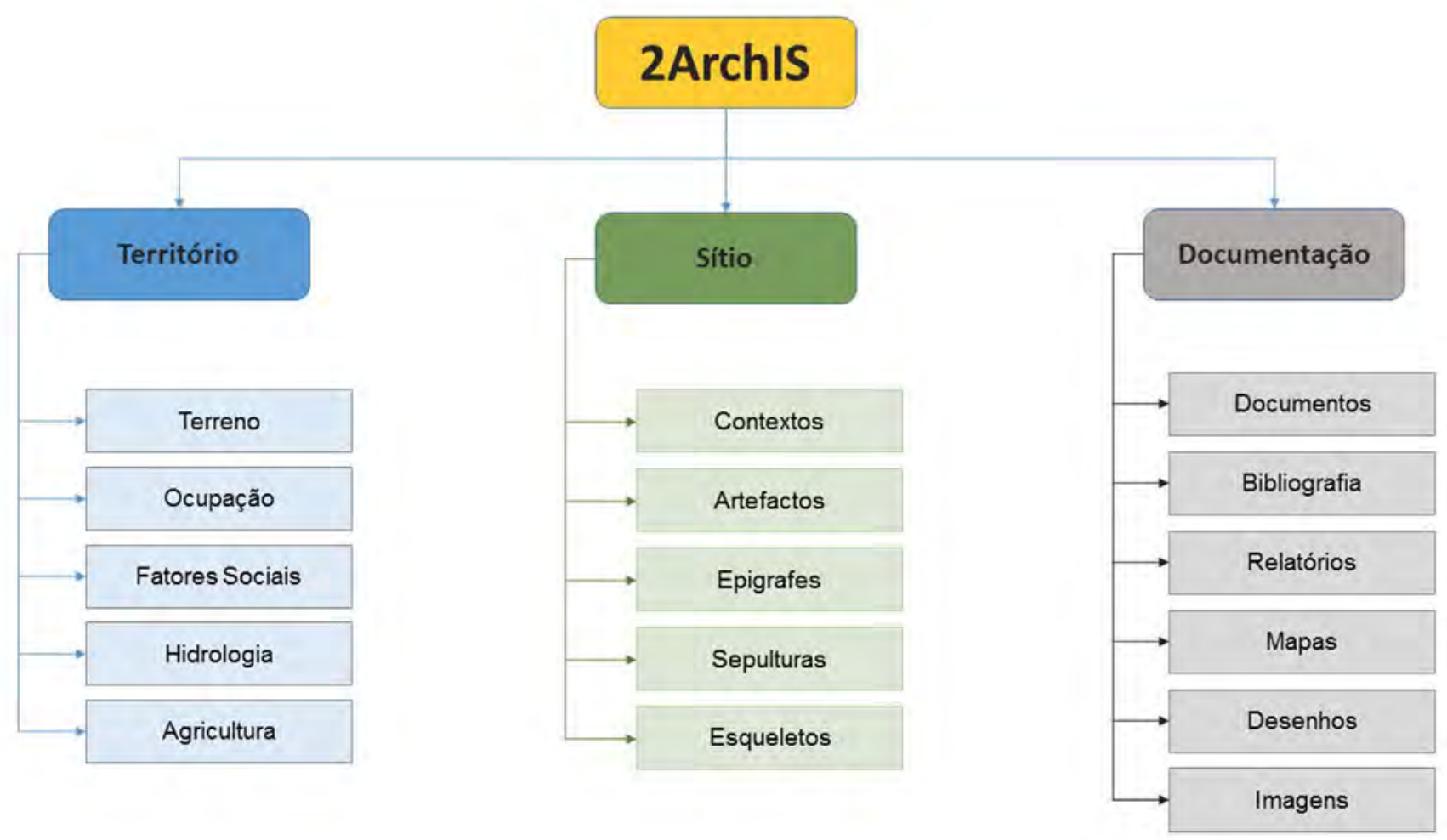

FIG. 2 Especificação da estrutura do sistema de informação 2ArchIS. 
Assim, através desta estrutura o sistema permite não só o acesso à informação por interrogação à base de dados, mas também através da representação gráfica da informação disponível para cada sítio em estudo, de acordo com critérios de interrogação estabelecidos. Detalharemos a seguir como foi estruturado o processo de recolha e registo dos dados e metadados.

\subsection{Recolha e armazenamento de informação}

Para aplicação desta metodologia, partimos do levantamento das várias fontes de informação, nomeadamente bibliográfica, cartográfica e fotográfica, bem como da informação disponível no Portal do Arqueó$\log 0^{3}$.

O sistema de Informação 2ArchIS estrutura a informação base dos sítios arqueológicos, associando-os com a(s) tipologia(s) dos sítios, período(s) cultural(ais), materiais resultantes das sondagens/escavações arqueológicas, contexto geográfico e cultural, de acordo com o formulário de registo apresentado na Figura 4.

Este sistema de informação usa como interface para registo de dados uma aplicação de back office,

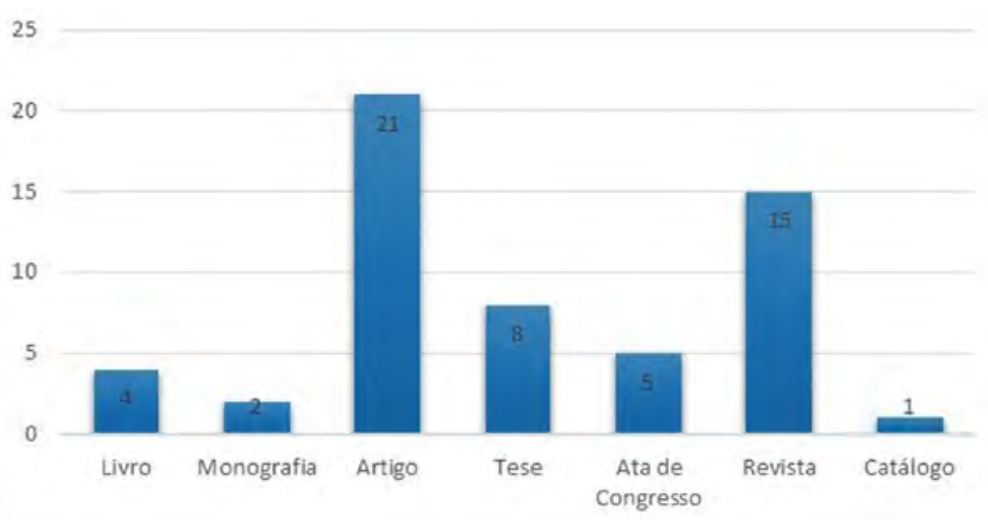

FIG. 3 Representação da amostra de dados bibliográficos recolhidos.

no qual pode ser inserida a informação pertinente para o projeto, com base em descritores normalizados. Esta aplicação foi desenvolvida com tecnologias open source de interface WEB (PHP e HTML), assente numa base de dados relacional MySQL e que permite a sua ligação direta a várias ferramentas de tratamento e de visualização de dados, bem como a interoperabilidade com estruturas externas (Botica 2017: 3). O requisito de ter uma aplicação de registo arqueológico com interface $W E B$, para utilização, quer em campo quer em

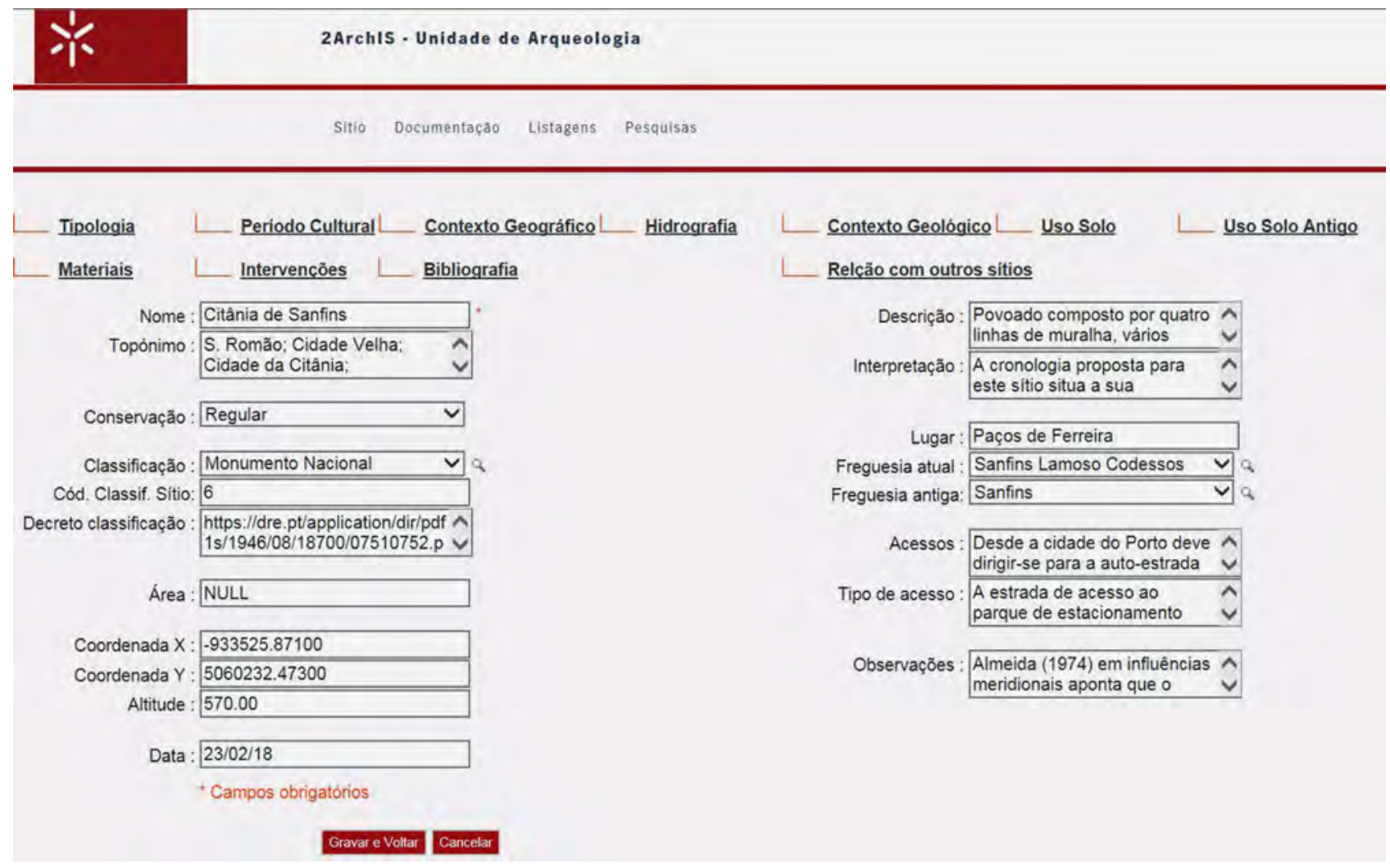

FIG. 4 Imagem de formulário de back office para registo de dados de sítio.

3 http://arqueologia.patrimoniocultural.pt/index.php?sid=sitios 


\section{ArchIS - Unidade de Arqueologia}

\section{Sítio Documentação Listagens Pesquisas}

\section{Associa a Sitio $\longleftarrow$ Associa a Glossário}

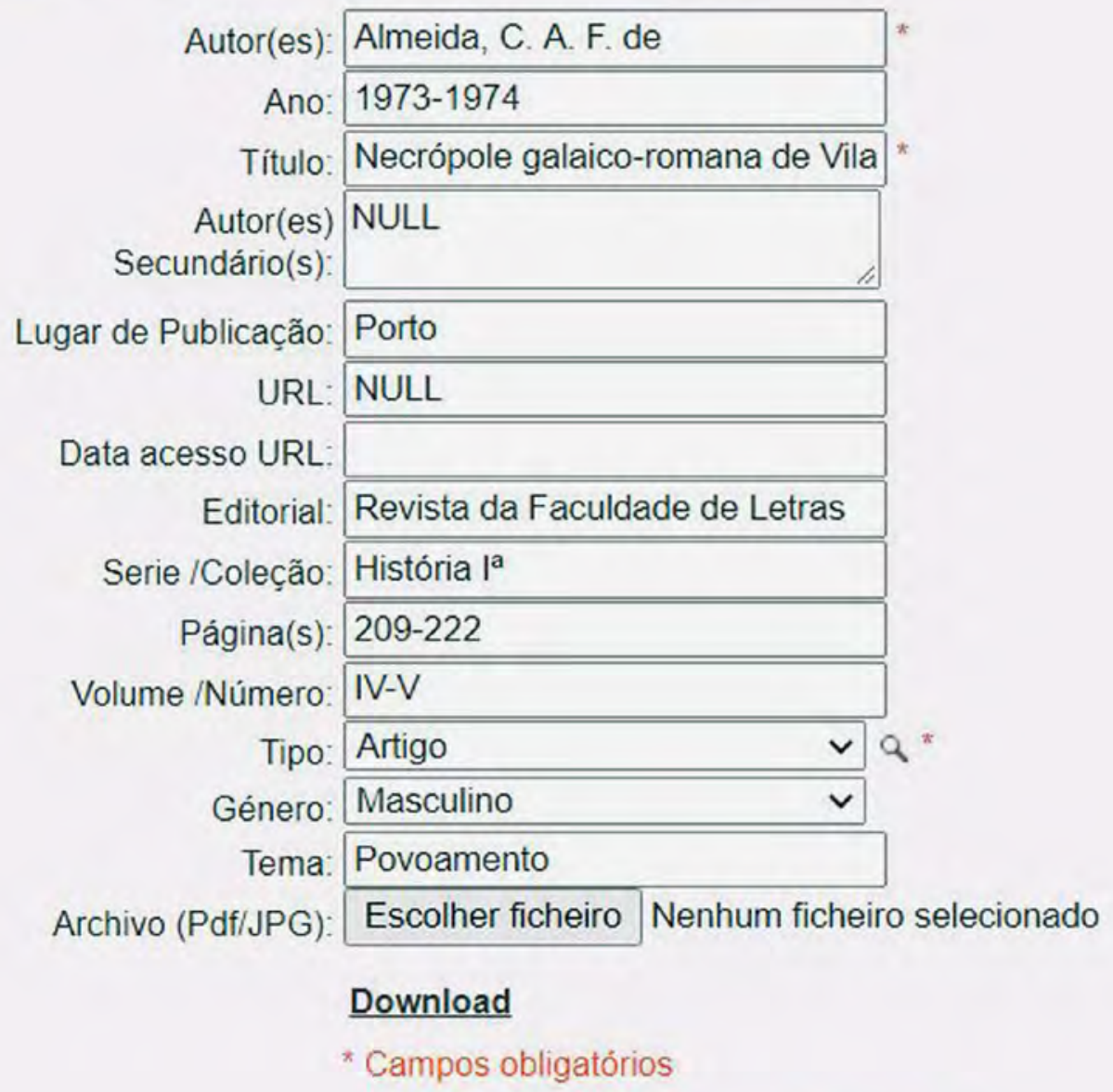

Guardar e Voltar Cancelar

FIG. 5 Imagem de formulário de back office para registo de bibliografia e associação a um ou mais sítios.

gabinete, implicou o seu desenvolvimento na UAUM, uma vez que não existia qualquer aplicação, com estas características, já desenvolvida por terceiros. O 2ArchIS é um sistema integrado modular que nos permite parametrizar as opções necessárias a cada projeto. É o caso das escavações arqueológicas, onde para além das opções disponíveis para este projeto, ainda se acrescentam os módulos de registo de estratigrafia, achados arqueológicos e estudo dos materiais. Esta interface permite a utilização da aplicação back office em qualquer dispositivo que possua um browser instalado (Explorer ou Chrome), isentando os seus utilizadores da instalação de qualquer software ou atualização de versões. Por outro lado, permite-nos disponibilizar de imediato, a todos os utilizadores, as alterações que vão sendo introduzidas, bem como novos módulos, como 
por exemplo os desenvolvidos na área da epigrafia, gliptografia, sepulturas e esqueletos.

A aplicação back office apresenta, para este projeto, um Menu Principal que, no nosso caso, é composto pelas entradas "Sítio", "Documentação", "Listagens" e "Pesquisa". Dentro da opção do menu para "Sítio" podemos encontrar o formulário de registo de sítio arqueológico, ligado a subformulários para associar o sítio a uma ou mais "Tipologia", "Período Cultural” e "Contexto Geográfico", entre outros. Na opção 'Documentação', incorporamos a bibliografia que está associada e interligada aos sítios, bem como as respetivas fotografias e a cartografia. A opção "Listagens", permite a visualização de toda a informação já integrada no sistema referente a cada sítio. Por fim, na opção "Pesquisa" encontramos um motor de busca de fácil acesso aos dados e metadados registados no sistema. Todos os registos estão georreferenciados e interligados entre si (Botica 2017: 4-5).

Ainda no que respeita à entrada "Documentação", podemos referir que nele integramos a bibliografia específica sobre os sítios em estudo (Figura 5), todas as referências e citações feitas sobre esses mesmos sítios, bem como as entradas encontradas no "Portal do Arqueólogo".

É no registo da documentação que associamos a cada entrada um ou mais sítios arqueológicos, bem como os metadados relativos ao tipo de dados que apresenta, nomeadamente se representam citações de trabalhos feitos no sítio arqueológico ou se apresentam dados sobre uma prospeção ou intervenção arqueológica. Neste caso, registam-se ainda metadados associados aos materiais que foram inventariados, bem como o seu tipo e caracterização.

\subsection{Mapear a bibliografia}

O mapeamento gráfico destes dados irá permitir ter uma visão mais abrangente e intuitiva sobre a quantidade e tipo de estudos feitos para determinado sítio, quais os sítios que foram mais e menos intervencionados, quais os sítios mais estudados e que documentos foram mais referenciados em estudos. Assim, a partir dos dados e metadados inseridos no sistema de informação, pode ser feito o mapeamento da informação bibliográfica, sobre as bases cartográficas selecionadas. A Figura 6 ilustra o mapeamento feito por sítio arqueológico e tipo de bibliografia (Estudo ou
Inventário), sendo que no caso dos estudos, os que estão assinalados com as circunferências mais pequenas representam 1 a 4 estudos referenciados para o sítio, as circunferências intermédias representam 5 a 12 estudos e as maiores 13 a 26 estudos, de acordo com a legenda apresentada. Como se pode verificar a maior parte dos sítios ou não foram estudados ou estão referenciados em menos de 4 estudos, destacando-se a Citânia de Sanfins como o sítio mais estudado. Também a representação dos inventários evidencia algumas assimetrias na sua distribuição, sendo que a maioria dos sítios tem apenas localizável 1 inventário.

Este mapeamento foi feito interligando o sistema 2ArchIS e um Sistema de Informação Geográfica (SIG), o ArcGIS, que permite projetar sobre diversas bases cartográficas um conjunto alargado de informação que, obedecendo a critérios de seleção estabelecidos, possibilita análises qualitativas e quantitativas aos dados, nomeadamente sobre a quantidade de estudos realizados sobre os sítios em análise; o tipo de dados existentes para os sítios e ainda as referências bibliográficas associadas aos sítios e a temas de investigação.

A visualização gráfica dos dados através do SIG, interligado com o 2ArchIS, põe em evidência a distribuição geográfica dos sítios e o nível de intervenção feita, em termos de tipos de intervenção, classificação, estado de conservação e de proteção. O mapa apresentado na Figura 7 coloca em evidência os sítios para os quais foi possível localizar dados relativos à caracterização dos sítios arqueológicos, materiais e estudos de povoamento, e os sítios para os quais esse tipo de dados não existem ou não estão localizáveis ou acessíveis. Podemos verificar que, de acordo com a legenda apresentada, a maior parte dos sítios arqueológicos aparece referenciada em estudos de povoamento e são poucos os sítios para os quais foi possível localizar e aceder a dados sobre escavações arqueológicas ou inventários de materiais.

Este tipo de análise foi muito importante para este trabalho porque, por um lado, nos permite fazer uma melhor avaliação sobre a quantidade e tipo de dados disponíveis e, por outro lado, permite elencar trabalhos a desenvolver futuramente para colmatar as lacunas detetadas. Este aspeto, tal como já referimos, será abordado nos trabalhos futuros que estamos a preparar. 


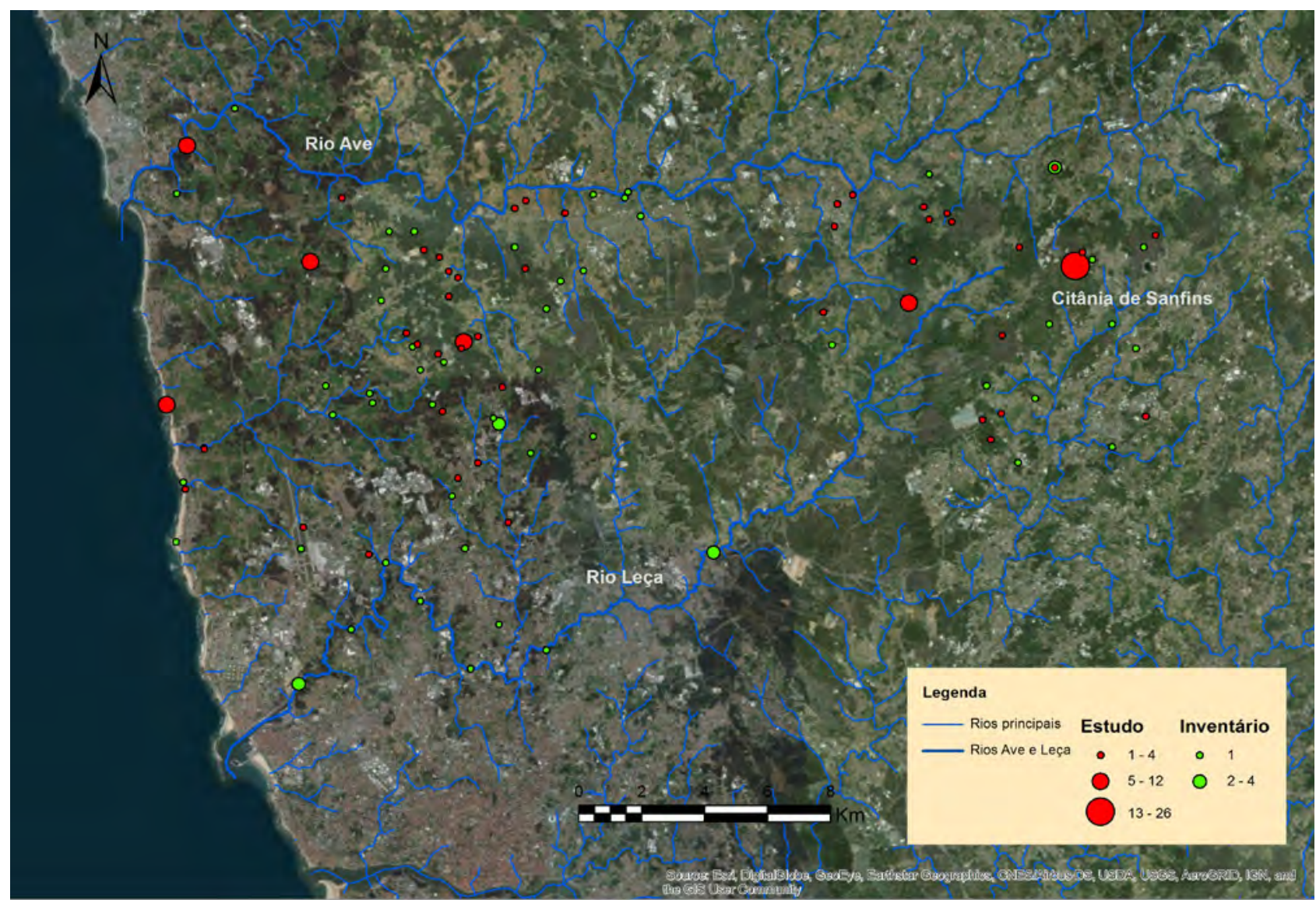

FIG. 6 Mapeamento dos inventários e estudos realizados sobre os sítios em análise.

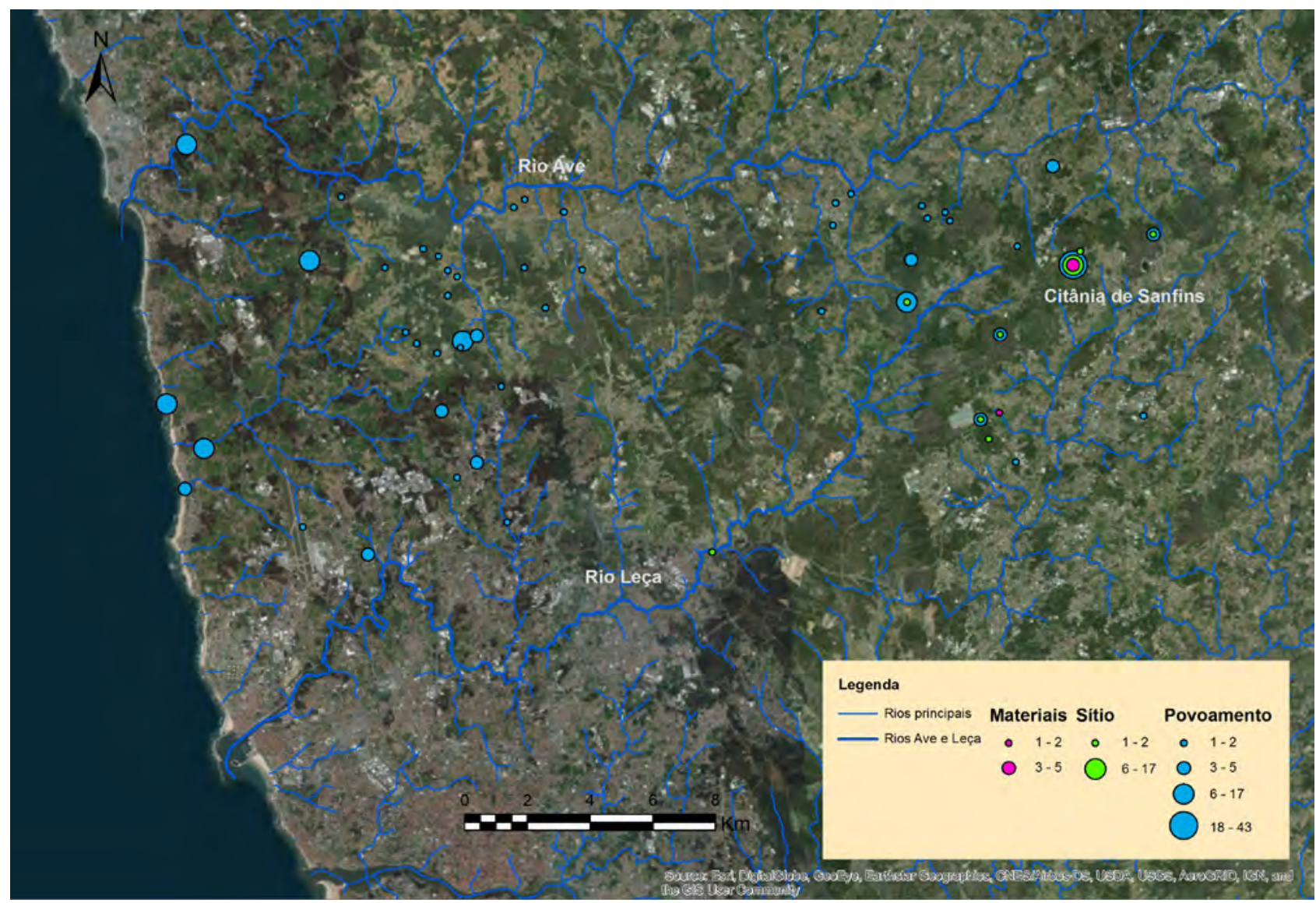

FIG. 7 Mapeamento da quantidade de inventários e estudos realizados para os sítios em análise. 


\section{CONSIDERAÇõES FINAIS}

A metodologia seguida no estudo da paisagem da Citânia de Sanfins tem por base o levantamento da informação existente sobre o território e sítios arqueológicos identificados. Como em qualquer projeto de investigação, também este está muito dependente da quantidade e qualidade dos dados que identificamos e recolhemos. A informação a que tivemos acesso estava muito dispersa, fragmentada e disponível em diferentes formatos. A reutilização dos dados localizados implicou um processo de recolha, sistematização e normalização, sem o qual não seria possível qualquer tipo de análise. Só após este processo de registo e normalização pudemos trabalhar no estudo da paisagem da Citânia de Sanfins, fazendo interrogações aos dados e projetando os resultados sobre diversas bases cartográficas. Estas representações gráficas da informação disponível, proporcionaram uma leitura abrangente da quantidade e especificidade dos estudos/dados referentes aos sítios arqueológicos que integram o projeto.

Este modelo de gestão e representação permitiu-nos ter uma visão geral dos estudos e projetos de investigação já realizados para os sítios em estudo, e identificar diferentes perspetivas de análise, reconhecendo as lacunas existentes e evidenciando necessidades de intervenção programada para colmatar essas lacunas.

A metodologia e estruturas de dados adotadas revelaram-se bastante eficazes para apoio ao estudo da paisagem da Citânia de Sanfins, destacando a necessidade de criação de um repositório de dados da arqueologia em Portugal, o qual iria reduzir o tempo consumido na recolha de dados e incrementar as possibilidades de reutilização em novos projetos de investigação.

\section{Referências bibliográficas}

ALMEIDA, M. (2017a) - De Augusta Emerita a Olisipo por Ebora: uma leitura do território a partir da rede viária. Lisboa.

ALMEIDA, M. (2017b) - Contributo para a normalização do registo de informação arqueológica a partir do estudo da via Emerita - Olisipo por Ebora. Ophiussa. 2, 167-184.

AREZES, A. - VARELA, J. (2017) - Castro de Guifões (Matosinhos) Das primeiras notícias aos resultados preliminares de um projecto de investigação. Arqueologia em Portugal. 2017 Estado da Questão, Associação dos Arqueólogos Portugueses. Lisboa, 125-136.

BOTICA, N. (2017) - Contributo do sistema de informação 2ArchIS para o conhecimento das cidades romanas de Braga e Lugo. Philtáte. Studia et acta antiquae Callaeciae. 2, 387-397.

BOTICA, N. - ANDRADE, F. - FONTES, L. (2016) - Georeferencing historical documents: the case of the Dume boundary. $9^{\text {th }}$ IADIS International Conference Information Systems. Lisboa, 125-129.

BOTICA, N. - MAGALHÃES, F. - MACHADO, D. - FONTES, L. (2021) Del sistema de información 2ArchIS al DataRepositóriUM: el estudio de caso de hallazgos arqueológicos. Cuadernos de Arqueología de la Universidad de Navarra. 29, 1-16. http://doi. org/10.15581/012.29.005.

BOTICA, N. - MARTINS, M. (2008) - Sistemas de informação em arqueologia - a experiência de Bracara Augusta. $10^{\circ}$ Congreso Internacional de Arqueoloxía de Vilalba. Vilalba, 11-14.

CLETO, J. - VARELA, J. (1999) - O Castro de Guifões (Matosinhos): dos estudos de Martins Sarmento às investigações da actualidade. Revista de Guimarães. Volume especial: II, 467-479.

DIVISÃO DE INVENTÁRIO DO INSTITUTO PORTUGUÊS DE ARQUEOLOGIA (2002) - Endovélico: Sistema de gestão e Informação Arqueológica. Revista Portuguesa de Arqueologia.

HOLLANDER, H., et al. (2019) - PARTHENOS Guidelines to FAIRify data management and make data reusable. http://doi. org/10.5281/zenodo.3368858.

MACIEL, S. (2019) - A paisagem na Idade do Ferro e Romanização da Citânia de Sanfins, Paços de Ferreira. Análise macro espacial. Tese de Mestrado. Universidade do Minho.

MARTINS, M. (1985) - Sondagens arqueológicas no Castro do Monte Padrão, em Santo Tirso. Cadernos de Arqueologia. Série II: 2, 217-230.

MOREIRA, A. (2005) - Estação Arqueológica Monte do Padrão. O Castro do Monte Padrão. Do Bronze Final ao fim da Idade Média. Santo Tirso.

MOREIRA, A. (2009) - Castellum Madiae. Formação e desenvolvimento de um "aglomerado urbano secundário" no ordenamento do povoamento romano entre o Leça e Ave. Tese de Doutoramento. Universidade de Santiago de Compostela.

NICCOLUCCI, F. - RICHARDS, J. D. (2013) - ARIADNE: Advanced Research Infrastructures for Archaeological Dataset Networking in Europe. International Journal of Humanities and Arts Computing. 7: 1-2, 70-88.

PINHO, J. (2009) - O 1. milénio A.C. e o establecimento rural romano na vertente fluvial do Ave: dinâmicas de establecimento sob o ponto de vista geo-espacial. Tese de Mestrado. Universidade de Lisboa.

RICHARDS, J. - WRIGHT, H. (2019) - COST 18128: SEADDA Management Committee.

SILVA, A. (2007) - A cultura castreja no norte de Portugal. Paços de Ferreira. 

AVALIADORES OPHIUSSA (2018-2020)

Adolfo Fernández Fernández

Ahmed Mcharek

Albert Ribera i Lacomba

Álvaro Gómez Peña

Amílcar Guerra

Ana Cristina Martins

Ana Delgado Hervás

Ana Margarida Arruda

Ana Maria Niveau de Villedary y Mariñas

António Faustino Carvalho

António Carlos Valera

Carlos P. Odriozola

Carlos Pereira

Cátia Mourão

Catarina Costeira

Catarina Viegas

Corina Liesau

Elisa Garcia Prosper

Elisa Guerra Doce

Enrique García Vargas

Ernst Pernicka

Esther Rodríguez González

Francisco B. Gomes

Francisco J. Núñez

F. Germán Rodríguez Martín

Javier Jiménez Ávila

Javier Mangado Llach

Jesús Acero Pérez

João Pedro Bernardes

João Senna-Martinez
João Luís Cardoso

Joaquina Soares

José Carlos Quaresma

José Clemente Martin de la Cruz

José Ruivo

Juan Antonio Cámara Serrano

Leonardo García Sanjuán

Luís Araújo

Luís Raposo

Macarena Bustamante

Maria João Valente

Maria José de Almeida

Mariano Torres Ortiz

Mário Jorge Barroca

Marta Moreno García

Mounir Fantar

Patrícia Matos

Raquel Vilaça

Ricardo Costeira da Silva

Roberto Risch

Rodrigo Banha da Silva

Rui Gomes Coelho

Rui Martiniano

Rui Morais

Sergio Escribano Ruiz

Sérgio Gomes

Simon Davis

Tomás Cordero Ruiz

Victor S. Gonçalves 


\section{POLÍTICA EDITORIAL}

A Ophiussa - Revista do Centro de Arqueologia da Universidade de Lisboa foi iniciada sob a direcção de Victor S. Gonçalves em 1996, tendo sido editado o volume 0. O volume 1 (2017) é uma edição impressa e digital da UNIARQ - Centro de Arqueologia da Universidade de Lisboa.

O principal objectivo desta revista é a publicação e divulgação de trabalhos com manifesto interesse, qualidade e rigor científico sobre temas de Pré-História e Arqueologia, sobretudo do território europeu e da bacia do Mediterrâneo.

A Ophiussa - Revista do Centro de Arqueologia da Universidade de Lisboa publicará um volume anual. A partir de 2018, os artigos submetidos serão sujeitos a um processo de avaliação por parte de revisores externos (peer review). O período de submissão de trabalhos decorrerá sempre no primeiro trimestre e a edição ocorrerá no último trimestre de cada ano.

A revista divide-se em duas secções: artigos científicos e recensões bibliográficas. Excepcionalmente poderão ser aceites textos de carácter introdutório, no âmbito de homenagens ou divulgações específicas, que não serão submetidos à avaliação por pares. Isentas desta avaliação estão também as recensões bibliográficas.

Todas as submissões serão avaliadas, em primeira instância, pela Coordenação Editorial, no que respeita ao seu conteúdo formal e à sua adequação face à política editorial e às normas de edição da revista. Os trabalhos que cumprirem estes requisitos serão posteriormente submetidos a um processo de avaliação por pares cega / blind peer review (mínimo de dois revisores). O Conselho Científico, constituído pela direcção da UNIARQ e por investigadores externos, acompanhará o processo de edição.

Esta etapa será concretizada por investigadores externos qualificados, sendo os respectivos pareceres entregues num período não superior a três meses. Os revisores procederão à avaliação de forma objectiva, tendo em vista a qualidade do conteúdo da revista; as suas críticas, sugestões e comentários serão, na medida do possível, construtivos, respeitando as capacidades intelectuais do(s) autor(es). Após a recepção dos pareceres, o(s) autor(es) tem um prazo máximo de um mês para proceder às alterações oportunas e reenviar o trabalho.

A aceitação ou recusa de artigos terá como únicos factores de ponderação a sua originalidade e qualidade científica. O processo de revisão é confidencial, estando assegurado o anonimato dos avaliadores e dos autores dos trabalhos, neste último caso até à data da sua publicação.

Os trabalhos só serão aceites para publicação a partir do momento em que se conclua o processo da revisão por pares. Os textos que não forem aceites serão devolvidos aos seus autores. O conteúdo dos trabalhos é da inteira respon- sabilidade do(s) autor(es) e não expressa a posição ou opinião do Conselho Científico ou da Coordenação Editorial. A Revista Ophiussa segue as orientações estabelecidas pelo Commitee on Publication Ethics (COPE, Comité de Ética em Publicações): https://publicationethics.org/.

O processo editorial decorrerá de forma objectiva, imparcial e anónima. Erros ou problemas detetados após a publicação serão investigados e, se comprovados, haverá lugar à publicação de correções, retratações e/ou respostas. As colaborações submetidas para publicação devem ser inéditas. As propostas de artigo não podem incluir qualquer problema de falsificação ou de plágio. Para efeito de detecção de plágio será utilizada a plataforma URKUNDU (https:// www.urkund.com/pt-br/).

As ilustrações que não sejam do(s) autor(es) devem indicar a sua procedência. O Conselho Científico e a Coordenação Editorial assumem que os autores solicitaram e receberam autorização para a reprodução dessas ilustrações, e, como tal, rejeitam a responsabilidade do uso não autorizado das ilustrações e das consequências legais por infracção de direitos de propriedade intelectual.

É assumido que todos os Autores fizeram uma contribuição relevante para a pesquisa reportada e concordam com o manuscrito submetido. Os Autores devem declarar de forma clara eventuais conflitos de interesse. As colaborações submetidas que, direta ou indiretamente, tiveram o apoio económico de terceiros, devem claramente declarar essas fontes de financiamento.

Os textos propostos para publicação devem ser inéditos e não deverão ter sido submetidos a qualquer outra revista ou edição electrónica. Aceitam-se trabalhos redigidos em português, inglês, espanhol, italiano e francês.

Esta edição disponibiliza de imediato e gratuitamente a totalidade dos seus conteúdos, em acesso aberto, de forma a promover, globalmente, a circulação e intercâmbio dos resultados da investigação científica e do conhecimento.

A publicação de textos na Ophiussa - Revista do Centro de Arqueologia da Universidade de Lisboa não implica o pagamento de qualquer taxa nem dá direito a qualquer remuneração económica.

Esta publicação dispõe de uma versão impressa, a preto e branco, com uma tiragem limitada, que será distribuída gratuitamente pelas bibliotecas e instituições mais relevantes internacionalmente, e intercambiada com publicações periódicas da mesma especialidade, que serão integradas na Biblioteca da Faculdade de Letras da Universidade de Lisboa. Conta, paralelamente, com uma versão digital, a cores, disponibilizada no endereço www.ophiussa.letras.ulisboa.pt, onde se pode consultar a totalidade da edição.

Para mais informações: ophiussa@letras.ulisboa.pt 


\section{EDITORIAL POLICY}

Ophiussa - Revista do Centro de Arqueologia da Universidade de Lisboa started in 1996, with the edition of volume 0 . From 2017, this journal is a printed and digital edition of UNIARQ - Centro de Arqueologia da Universidade de Lisboa.

The main objective of this journal is the publication and dissemination of papers of interest, quality and scientific rigor concerning Prehistory and Archeology, mostly from Europe and the Mediterranean basin.

Ophiussa - Revista do Centro de Arqueologia da Universidade de Lisboa will publish an annual volume. From 2018, submitted articles will be subject to a peer-review evaluation process. The submission period will always occur in the first quarter of each year and the edition will occur in the last quarter.

The journal is divided into two sections: scientific articles and bibliographic reviews. Exceptionally, texts of an introductory nature may be accepted, in the context of specific tributes or divulgations, which will not be submitted to peer-review evaluation. Exemptions from this evaluation are also the bibliographic reviews.

All submissions will be considered, in the first instance, by the Editorial Board, regarding its formal content and adequacy in face of the editorial policy and the journal's editing standards. Papers that meet these requirements will subsequently be submitted to a blind peerreview process (minimum of two reviewers). The Scientific Council, constituted by the directors of UNIARQ and external researchers, will follow the editing process.

This stage will be carried out by qualified external researchers, and their feedback will be delivered within a period of no more than two months. The reviewers will carry out the evaluation in an objective manner, in view of the quality and content of the journal; their criticisms, suggestions and comments will be, as far as possible, constructive, respecting the intellectual abilities of the author(s). After receiving the feedback, the author(s) has a maximum period of one month to make the necessary changes and resubmit the work.

Acceptance or refusal of articles will have as sole factors of consideration their originality and scientific quality. The review process is confidential, with the anonymity of the evaluators and authors of the works being ensured, in the latter case up to the date of its publication.

Papers will only be accepted for publication as soon as the peer review process is completed. Texts that are not accepted will be returned to their authors. The content of the works is entirely the responsibility of the author(s) and does not express the position or opinion of the Scientific Council or Editorial Board. The Journal Ophiussa follows the guidelines established by the Commitee on Publication Ethics (COPE, the Ethics Committee Publications): https:// publicationethics.org/
The editorial process will be conducted objectively, impartially and anonymously. Errors or problems detected after publication will be investigated and, if proven, corrections, retractions and / or responses will be published. Contributions submitted for publication must be unpublished. Article submissions can not include any problem of forgery or plagiarism. In order to detect plagiarism, the URKUNDU platform will be used.

Illustrations that are not from the author(s) must indicate their origin. The Scientific Council and Editorial Board assume that the authors have requested and received permission to reproduce these illustrations and, as such, reject the responsibility for the unauthorized use of the illustrations and legal consequences for infringement of intellectual property rights.

It is assumed that all Authors have made a relevant contribution to the reported research and agree with the manuscript submitted. Authors must clearly state any conflicts of interest. Collaborations submitted that directly or indirectly had the financial support of third parties must clearly state these sources of funding.

Texts proposed for publication must be unpublished and should not have been submitted to any other journal or electronic edition. Works written in Portuguese, English, Spanish, Italian and French are accepted.

The publication of texts in Ophiussa - Revista do Centro de Arqueologia da Universidade de Lisboa does not imply the payment of any fee nor does it entitle to any economic remuneration.

This edition immediately and freely provides all of its content, in open access, in order to promote global circulation and exchange of scientific research and knowledge. This publication has a limited printed edition in black and white, which will be distributed free of charge by the most relevant international libraries and institutions, and exchanged with periodicals of the same specialty, which will be integrated in the Library of Faculdade de Letras of Universidade de Lisboa. It also has a digital version, in color, available at address http://ophiussa.letras.ulisboa.pt, where one can consult the entire edition.

For more information contact: ophiussa@letras.ulisboa.pt 
REVISTA DO CENTRO DE ARQUEOLOGIA DA UNIVERSIDADE DE LISBOA

\section{$4-2020$}

ÍNDICE

The megalithic clusters of Deserto and Barrocal das Freiras

(Montemor-o-Novo, Middle Alentejo) in the building of the sacred

landscapes of ancient peasant societies of the $4^{\text {th }}$ and $3^{\text {rd }}$ millennia BCE

VICTOR S. GONÇALVES, MARCO ANTÓNIO ANDRADE

From aDNA to Archaeology: Genética da transição Calcolítico-Idade do Bronze

no Sul de Portugal

ANA CATARINA BASÍLIO

Foundry in the Late Bronze Age Baiões/Santa Luzia Cultural Group:

some reflections starting from a new metallic mould for unifacial palstaves

JOÃO CARLOS SENNA-MARTINEZ, PEDRO VALÉRIO, MARIA HELENA CASIMIRO,

LUÍS M. FERREIRA, MARIA DE FÁTIMA ARAÚJO, HORÁCIO PEIXOTO

O conjunto vítreo da necrópole da I Idade do Ferro da Fonte Velha

de Bensafrim (Lagos)

FRANCISCO B. GOMES

Espacios de almacenamiento y producción de la Turdetania.

Una reflexión más allá de los hornos

VIOLETA MORENO MEGÍAS

Os três sarcófagos etruscos da colecção de Sir Francis Cook

no Museu Arqueológico de São Miguel de Odrinhas (Sintra)

MARTA RIBEIRO, NUNO SIMÕES RODRIGUES

Nuevos datos para el conocimiento del suburbium del Municipum

Florentinum Iliberritanum (Granada): las recientes intervenciones

en el solar de Mondragones

ÁNGEL RODRÍGUEZ AGUILERA, MACARENA BUSTAMANTE-ÁLVAREZ,

JULIA RODRÍGUEZ AGUILERA, CARMEN JÓDAR HÓDAR, JOSÉ M. GARCÍA-CONSUEGRA FLORES

Mapear a bibliografia. Abordagem metodológica para a gestão

187

de dados bibliográficos

NATÁLIA BOTICA, SÍLVIA MACIEL, REBECA BLANCO-ROTEA

Recensões bibliográficas

(TEXTOS: JORGE DEL REGUERO GONZÁLEZ, LUIS MIGUEL CARRANZA PECO,

MARTA BERMÚDEZ CORDERO, ÁLVARO GÓMEZ PEÑA, ANDRÉ TEXUGO)

Da CAALG à UNIARQ: a génese do Centro de Arqueologia da Universidade

de Lisboa no quadro do sistema científico de meados dos anos 70

a meados dos anos 90 do século XX

ANDRÉ PEREIRA

Avaliadores Ophiussa (2018-2020) 